\title{
DIGITAL COLOUR MAPPING OF THE SKY FROM SUPERCOSMOS
}

\author{
H.T. MacGILLIVRAY \\ Royal Observatory Edinburgh, UK
}

\section{Introduction}

The major photographic surveys of the sky (undertaken with the ESO, Palomar and UK Schmidt telescopes) still hold enormous potential regarding the information content of the original plate material. The problem to date has been to extract that information in a meaningful and quantitative way, hence giving rise to the large number of digitisation programmes currently underway and which are reported elsewhere in this volume.

Until recently, such digitisation efforts have resulted solely in the production of monochrome images of the sky. Now, however, with the SuperCOSMOS scanning programme (aimed at systematically digitising these survey material) we have succeeded in combining the monochrome images from the separate wavebands in order to produce true colour digital images (an area which has for long been exclusively the domain of photography).

\section{Method and Applications}

The method is straightforward and directly similar to the photographic equivalent: digital images from blue, green and red plates are combined to produce the final colour-composited digital image (see Plate 1). Unfortunately, for much of the sky, 'green' plates are not available. However, sensible colours are still achieved using the blue and red data alone.

There are many advantages to having available a digital colour map of the sky. Among them: (i) the colours can be optimised and calibrated; (ii) the images are directly amenable to computer manipulation (pan, zoom, unsharp masking, contrast enhancement, etc.; (iv) the colours (in their own 
right) highlight interesting features worthy of further follow-up study; (iv) interesting objects (e.g., quasars, brown dwarfs) are readily detected.

We plan to distribute the SuperCOSMOS colour maps on CD-ROM. 


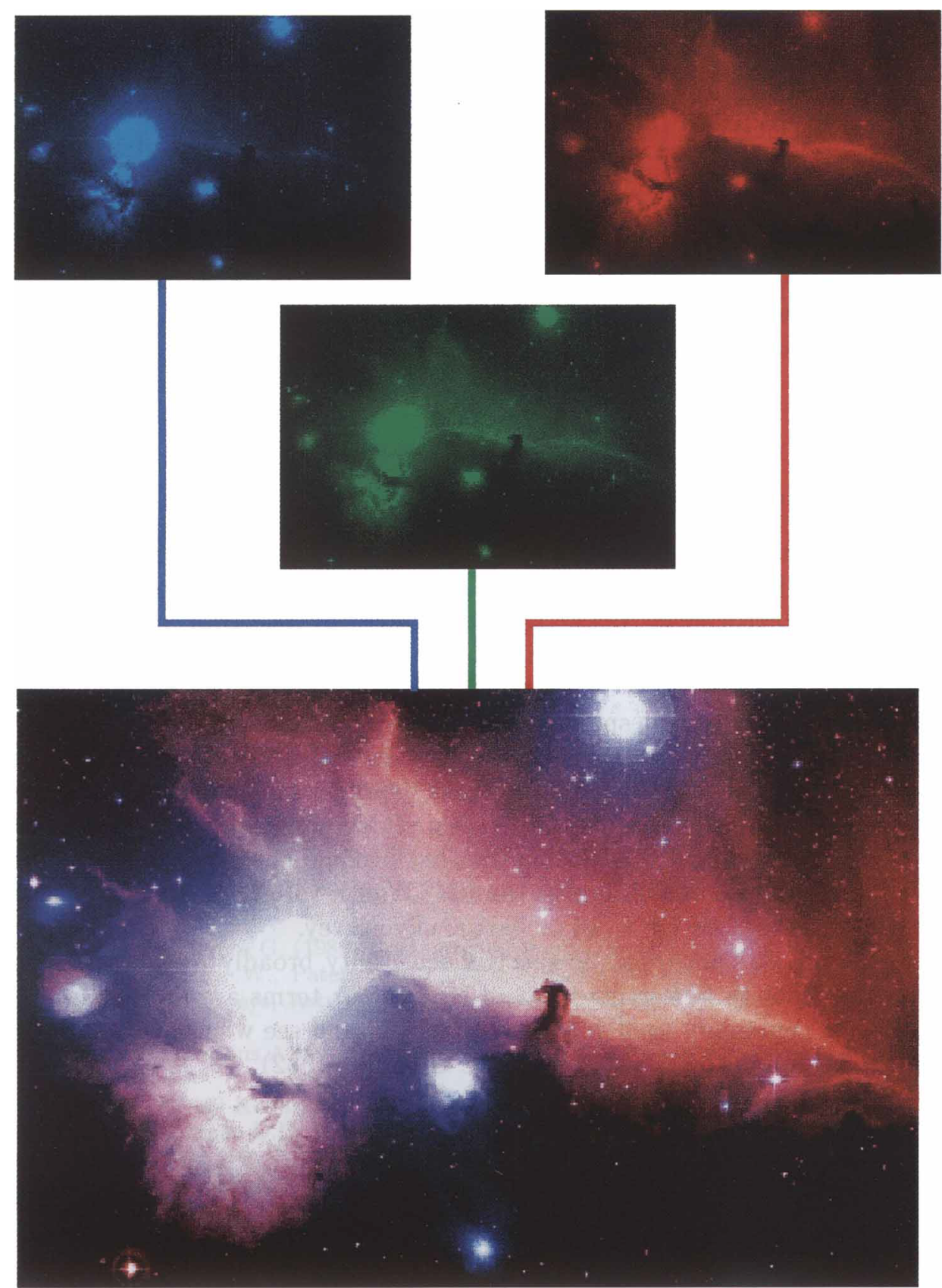

Figure 1. Illustrating the superposition of digital images from the blue, green and red plate data to produce the final colour digital image. 